\title{
Deteç̧ão de exsudatos em imagens de retina por técnicas de morfologia matemática e agrupamento nebuloso
}

Rodrigo de Melo Souza Veras*, Fátima Nelsizeuma Sombra de Medeiros, Flávio Henrique Duarte de Araújo, André Macêdo Santana, Romuere Rodrigues Veloso e Silva

Resumo A retinopatia diabética (RD) é uma das principais complicações do diabetes mellitus, pois causa sérios danos à retina e consequentemente à visão, podendo inclusive resultar em cegueira. O diagnóstico da RD é realizado através da análise visual de imagens de retina, sendo os exsudatos (depósitos de gordura) os principais padrões rastreados pelo médico especialista. Vale destacar que o diagnóstico precoce, realizado através do monitoramento regular, associado ao tratamento adequado apresenta inúmeros benefícios na prevenção da deficiência visual. Neste trabalho, é proposto um algoritmo de detecção de exsudatos em imagens de retina, cuja validação experimental é realizada na base pública DIARETDB1. A escolha desta base se deve à disponibilidade da localização dos exsudatos na retina, o que constitui o padrão ouro para a validação dos algoritmos. A metodologia proposta combina agrupamento nebuloso e técnicas de morfologia matemática, além de prover a detecção do disco óptico considerando que o mesmo é um ponto de convergência dos vasos. Os resultados mostraram que o método de detecção de exsudatos apresentou taxas de acerto na avaliação por imagens e por regiões na ordem de $73,03 \%$ e $99,41 \%$, respectivamente. Estes resultados confirmam que houve uma melhoria no desempenho na detecção, quando comparados, aos resultados de métodos disponíveis na literatura.

Palavras-chave Retinopatia diabética, Imagens de retina, Detecção de exsudatos.

\section{Exudate detection in retina images by mathematical morphology techniques and fuzzy clustering}

Abstract Diabetic retinopathy (DR) is one of the major complications of diabetes mellitus and, furthermore it causes severe damage to the retina and consequently to the vision. DR may lead to blindness and therefore it is important to prevent it or early detect and treat it. The diagnosis of DR is performed by visual analysis of retinal images being exudates (fat deposits) the main patterns traced by a specialist doctor. It is noteworthy that early diagnosis, through regular monitoring when coupled with proper treatment, results in numerous benefits in the prevention of visual impairment. Thus, this paper proposes an algorithm for exudate detection in retinal images, whose experimental validation is performed on retina images of the publicly available DIARETDB1 database. The reason for choosing this database is that it provides spatial coordinates of exudates in retina images which constitute ground truths for the algorithm validation. The proposed methodology combines fuzzy clustering and mathematical morphology techniques, and thus it provides a method for optic disk detection considering that it is as the convergent point of vessels. The exudate detection method presented successful rates of $73.03 \%$ and $99.41 \%$ concerning the use of the whole image and only partial regions, respectively. These results confirm the performance improvement provided by the proposed methodology, when comparing it to other methods available in the literature.

Keywords Diabetic retinopathy, Retinal images, Exudates detection. 


\section{Introdução}

Imagens digitais de fundo de olho podem prover importantes informações sobre mudanças causadas por doenças oculares locais e sinais recentes de doenças sistêmicas como hipertensão, arteriosclerose e diabetes mellitus (Walter et al., 2002). A retina constitui a membrana mais interna do olho, situando-se na sua parede posterior conforme ilustra a Figura 1.

A identificação de doenças do olho humano através de técnicas de processamento digital de imagens (PDI) vem sendo utilizada cada vez mais em aplicações de detecção de alterações estruturais na retina e de doenças como a Retinopatia Diabética (RD), Edema Macular (EM) e Glaucoma. A RD é uma complicação do diabetes mellitus e é a maior causadora de perda de visão em diabéticos (Sopharak et al., 2010). Esta doença, assintomática na fase inicial, é de difícil identificação e resulta de alterações vasculares na retina, causando inchaços de capilares conhecidos como microaneurismas (MA). Vale ressaltar que os MAs são de difícil visualização, devido às pequenas dimensões $(0,1 \mathrm{~cm})$ dos mesmos. Eventualmente, estas estruturas podem se romper e constituir fontes de extravasamento de plasma causando o espessamento da retina, ou edema, e, quando ocorrem na região macular, podem causar perda de visão (Giancardo et al., 2012).

O monitoramento contínuo da RD é de suma importância para que a doença seja diagnosticada em seu estágio inicial ou mesmo antes do aparecimento dos primeiros sintomas e assim o tratamento precoce possa evitar ou reduzir o grau de perda da visão do paciente. $\mathrm{O}$ diagnóstico da $\mathrm{RD}$ é feito por exame visual clínico por um médico oftalmologista ou profissional treinado. A precisão do diagnóstico depende de

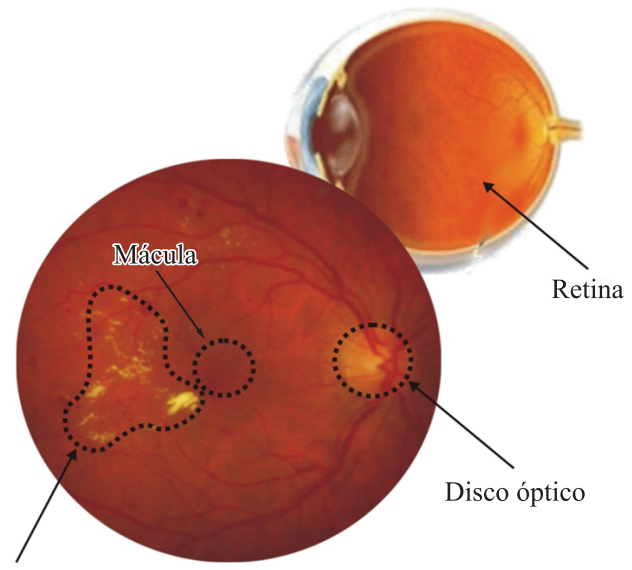

Exsudatos

Figura 1. Elementos característicos do olho e da retina. Figure 1.Components of the human eye and retina. fatores como a qualidade da imagem, habilidade e experiência do especialista, dentre outros. Assim, um sistema automático de auxílio ao diagnóstico de doenças do sistema visual, através de imagens de retina, pode reduzir significativamente a carga de trabalho do médico, particularmente em serviços de triagem de pacientes.

Neste artigo, as estruturas da retina em estudo são o disco óptico (DO) e os exsudatos, sendo estes últimos áreas de depósitos de gordura na parede da retina e sinais clínicos de RD. Eles geralmente formam aglomerados que podem estar distribuídos em toda a retina (Sopharak et al., 2010) e são classificados como duros e moles. Os exsudatos duros aparecem como regiões de cor amarelo brilhante e podem apresentar formatos distintos, com bordas bem definidas. Os exsudatos moles também são conhecidos como manchas algodonosas (semelhantes a pedaços de algodão), não possuem bordas definidas e aparecem em estágios mais avançados da RD (Basha e Prasad, 2008).

O objetivo deste artigo é propor algoritmos robustos para detecção de exsudatos, os quais possam ser utilizados no suporte à decisão clínica, contribuindo assim para a melhoria da qualidade do diagnóstico e redução da carga de trabalho do profissional. Quando aplicado em triagem de pacientes, a imagem da retina pode ser segmentada e avaliada, inicialmente pelo sistema automático. Na sequência, o profissional médico deve avaliá-la com maior detalhamento e encaminhar o pacientes a centros especializados, quando for o caso.

\section{Estado da arte}

Vários métodos de detecção de exsudatos (Basha e Prasad, 2008; García et al., 2009; Ram e Sivaswamy, 2009; Sopharak et al., 2010) foram propostos e existem, basicamente, três estratégias de abordagem do problema. Algumas estratégias envolvem limiarização e crescimento de região (García et al., 2009), outras utilizam técnicas de agrupamentos (Ram e Sivaswamy, 2009) e os demais algoritmos baseiam-se na reconstrução morfológica e em características de cor e forma dos exsudatos (Basha e Prasad, 2008).

A proposta de Sinthanayothin et al. (2002) detectou os candidatos a exsudatos utilizando técnica de segmentação baseada em crescimento de região recursivo e limiar adaptativo. Similarmente, Usher et al. (2004) utilizaram o mesmo recurso para a extração dos candidatos a exsudatos, porém classificaram os candidatos extraídos com uma rede neural multicamadas (MLP-Multilayer Perceptron). García et al. (2009) e Kavitha e Shenbaga (2005) aplicaram limiarização de vários níveis para segmentar o DO e exsudatos. Em Kavitha e Shenbaga (2005) o 
DO foi detectado pela convergência dos vasos e em García et al. (2009) foi realizada uma classificação com MLP e máquina de vetor de suporte (SVM - Support Vector Machine).

A estratégia de utilizar técnicas de limiarização aliadas ao crescimento de região é, a princípio, simples e de fácil implementação. Contudo, imagens de baixa qualidade afetam o resultado da separação de lesões brilhantes e escuras pelo processo de limiarização, assim como a extração das regiões de exsudatos por técnicas de crescimento de região. Esse fato ocorre porque parâmetros como a seleção dos valores de limiar, pontos de origem (semente) da região e critério de parada são difíceis de serem estabelecidos automaticamente.

Osareh et al. (2001), Sopharak et al. (2009) e Zhang e Chutatape (2005) propuseram o uso do algoritmo $K$-médias nebuloso para segmentar a retina em grupos de pixels com cores semelhantes. Em (Osareh et al., 2001) os autores utilizaram o espaço de cor RGB e em Zhang e Chutatape (2005) os autores utilizaram o sistema Luv (Gonzalez e Woods, 2010). Sopharak et al. (2009) utilizaram, em seu trabalho, quatro atributos de entrada para o algoritmo $K$-médias nebuloso, a saber, valor de intensidade, desvio padrão da intensidade, valor de matiz e o número de pixels de borda em uma imagem de bordas. Além disso, os mesmos autores aplicaram uma reconstrução morfológica por dilatação como pós-processamento. É importante destacar que as técnicas de agrupamento utilizadas na detecção de exsudatos dependem do conjunto de atributos de entrada, do método de agrupamento e da definição do número de classes a ser utilizado.

Uma estratégia comumente utilizada na detecção de exsudatos é a busca por regiões na imagem, que possuam características similares às dessas estruturas (ex. forma, cor, brilho, etc.). Walter et al. (2002) aplicaram técnicas de Morfologia Matemática (MM), particularmente a reconstrução matemática para detectar contornos típicos de exsudatos. Utilizando abordagem similar, Katarzyna et al. (2004) empregaram a técnica de watershed (Soille, 2003) com marcador automático. Basha e Prasad (2008) associaram técnicas de MM à lógica nebulosa para segmentar a imagem em regiões e posteriormente classificá-las em regiões de exsudatos ou não-exsudatos. Dado que as técnicas de reconstrução morfológica se mostraram sensíveis à variação de contraste das imagens e à iluminação não uniforme, a detecção dos exsudatos não foi bem sucedida, requerendo, portanto, um tratamento prévio no ajuste de iluminação, por exemplo.

Mais recentemente Sopharak et al. (2010) utilizaram uma SVM para realizar a classificação pixel a pixel da imagem, identificando aqueles pertencentes ou não a uma região de exsudatos. Apesar dos resultados bem sucedidos relatados, a utilização de classificadores (neste e em outros trabalhos citados anteriormente) exige um alto poder computacional para os processos de treinamento e classificação. Köse et al. (2012) se basearam no fato de que o fundo de imagens de retina saudáveis possui padrões regulares de cor e textura e com isso, estimaram o plano de fundo ótimo da imagem e os demais padrões foram considerados anormais. $\mathrm{O}$ método foi capaz de identificar a existência ou não de exsudatos, para confirmar se o paciente possui RD. Entretanto, Köse et al. (2012) não estavam interessados, neste artigo, na detecção dos exsudatos. Vale observar que o processo de determinação do plano de fundo ótimo se mostra sensível à iluminação não uniforme e ainda depende de resultados da detecção do DO e da rede de vasos.

Este trabalho se baseia no modelo introduzido por Ram e Sivaswamy (2009) para desenvolvimento da metodologia. Em seu trabalho, Ram e Sivaswamy (2009) propuseram um método de agrupamento em múltiplos espaços de cores, sendo que o agrupamento foi realizado em dois espaços de atributos diferentes e combinados para fins de segmentação dos exsudatos. O processo de agrupamento foi capaz de segmentar as regiões de exsudatos, porém, ao final da execução do algoritmo verificou-se que a taxa de acerto poderia ser melhorada, caso fosse aplicado um pós-processamento da imagem para remoção dos falsos candidatos.

\section{Materiais e Métodos}

\section{Base de imagens}

A base de imagens utilizada para o teste dos algoritmos foi a DIARETDB $1{ }^{1}$ que é composta por um conjunto de 89 imagens (Kauppi et al., 2007). As conhecidas bases de imagens de retina DRIVE ${ }^{2}, \mathrm{STARE}^{3}, \mathrm{MESSIDOR}^{4}$ e ARIA $^{5}$ não apresentam, informações acerca da presença ou ausência de exsudatos. Diferentemente, a base DIARETDB1 é bastante utilizada em trabalhos de detecção de exsudatos (Giancardo et al., 2012), porque possui a marcação de achados relacionados a quatro patologias: hemorragias, exsudatos moles, exsudatos duros e pontos vermelhos.

A marcação das patologias foi realizada na referida base por quatro especialistas e em algumas imagens, não houve consenso entre todos. Neste trabalho, são considerados exsudatos apenas as regiões marcadas por três dos quatro especialistas, como sugerem os próprios autores da base. A Figura 2 apresenta

${ }^{1}$ Disponível em: $<$ http://www.lut.it.fi/project/imageret $>$

${ }^{2}$ Disponível em: $<$ http://www.isi.uu.nl/Research/Databases/DRIVE/>

${ }^{3}$ Disponível em: < http://www.ces.clemson.edu/ ahoover/stare/>

${ }^{4}$ Disponível em: $<$ http://messidor.crihan.fr/index-en.php $>$

${ }^{5}$ Disponível em: <http://www.eyecharity.com/aria_online/> 
exemplos de imagens da base DIARETDB1, em que a Figura $2 \mathrm{c}$ apresenta as marcações das regiões de exsudatos realizadas por 4 especialistas. Quanto mais claro é o pixel na imagem, maior é o grau de concordância entre os diagnósticos. Regiões com cor branca representam áreas onde houve $100 \%$ de concordância entre os especialistas.

\section{Algoritmo de Ram e Sivaswamy}

A metodologia de detecção automática de exsudatos proposta no presente artigo tomou por base o trabalho de Ram e Sivaswamy (2009) que segue basicamente as três etapas apresentadas no diagrama da Figura 3 e descritas a seguir.

\section{Agrupamento de pixels}

Nesta etapa cada imagem da retina, que estava no modelo RGB, foi convertida para outros três espaços de cores: Luv (L se refere à luminância, u e v se referem à crominância), HSV (hue, saturation, value - matiz, saturação, valor), HSI (hue, saturation, intensity - matiz, saturação, intensidade). Maiores detalhes sobre espaços de cor podem sem encontrados em (Gonzalez e Woods, 2010). A partir desses espaços de cores são formados dois vetores de atributos (features) $f_{1}=(\mathrm{H}, \mathrm{S}, \mathrm{V}, \mathrm{I})$ e $f_{2}=(\mathrm{R}, \mathrm{G}, \mathrm{L}, \mathrm{u}, \mathrm{v})$ os quais são usados como entradas do algoritmo de agrupamento $K$-médias (Bezdek et al., 1993).
$\mathrm{O}$ algoritmo $K$-médias utiliza um número fixo de grupos iniciais determinado pelo usuário, de modo a particionar $n$ objetos do conjunto de dados em $K$ agrupamentos, otimizando uma função objetivo que é geralmente representada pelo somatório das distâncias entre os objetos e o centróide que o rotula. A saída deste algoritmo consiste de duas imagens $\left(I_{1}\right.$ e $\left.I_{2}\right)$ que são, respectivamente, os resultados do agrupamento segundo os vetores $f_{1}$ e $f_{2}$.

A imagem resultante do agrupamento do vetor de atributos $f_{1}$ corresponde à segmentação das seguintes regiões da retina: lesões brilhantes e plano de fundo brilhante, vasos, plano de fundo escuro e mácula, plano de fundo geral da retina e regiões periféricas. No caso do vetor de atributos $f_{2}$, o resultado da segmentação é composto pelo DO e exsudatos duros, vasos, lesões escuras e plano de fundo escuro, regiões em torno de objetos luminosos e outros pixels de plano de fundo.

\section{Seleção dos agrupamentos}

A partir das imagens $I_{1}$ e $I_{2}$ (imagens semelhantes às utilizadas por Ram e Sivaswamy são apresentadas nas Figura 8b, c, respectivamente) foram selecionados grupos que representam as regiões de exsudatos e DO. Na imagem $I_{l}$ o objetivo é determinar o grupo correspondente a lesões brilhantes e plano de fundo brilhante. Dado que essas regiões são as mais brilhantes da imagem original, foi selecionado
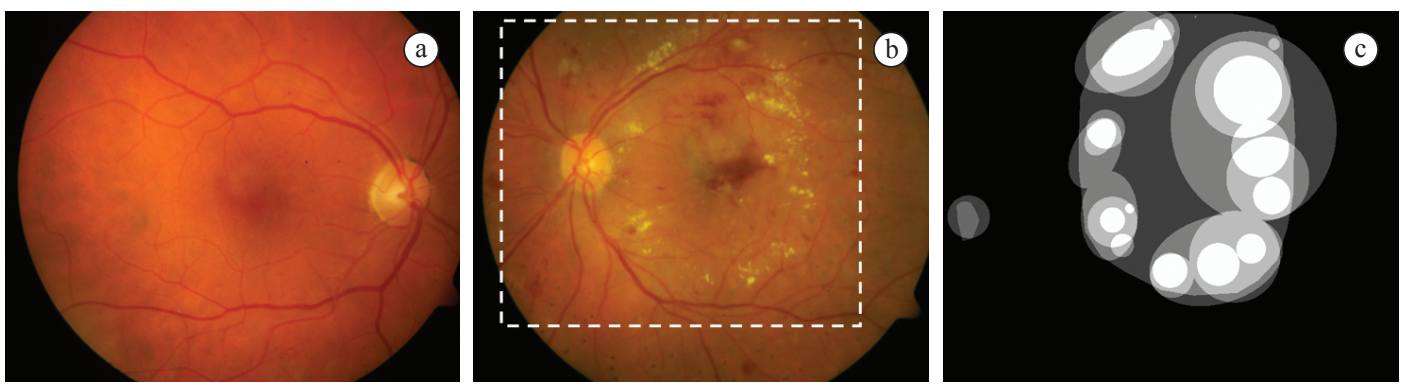

Figura 2. Exemplos de imagens da base DIARETDB1: a) retina saudável, b) retina com exsudatos e hemorragias, c) marcação dos exsudatos presentes na imagem da Figura $2 \mathrm{~b}$.

Figure 2. Samples of DIARETDB1 image database: a) healthy retina, b) retina with serveral exudates and bleeding, c) gold standard image for Figure $2 b$.

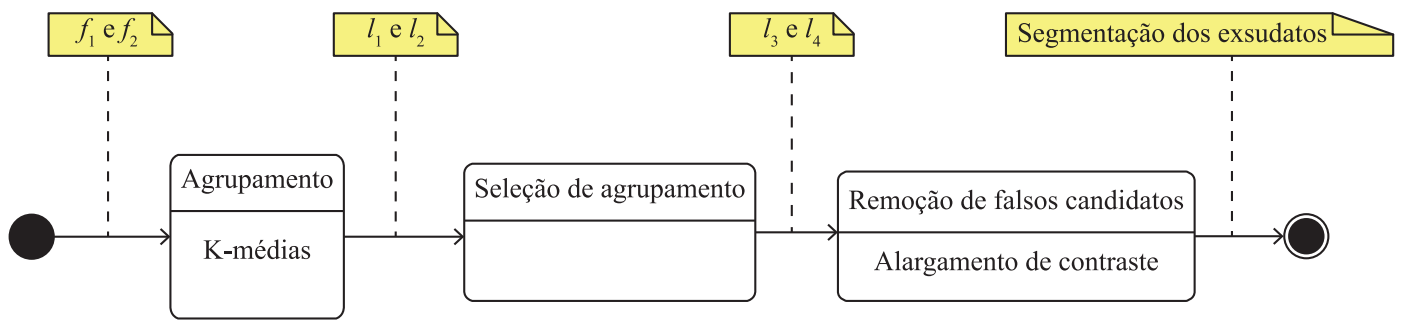

Figura 3. Diagrama do algoritmo introduzido por Ram e Sivaswamy (2009).

Figure 3. Diagram of the algorithm introduced by Ram and Sivaswamy (2009). 
aquele agrupamento de maior intensidade I no espaço HSI. Na imagem $I_{2}$ o grupo selecionado é aquele que corresponde ao DO e exsudatos duros. Como essas regiões exibem uma cor amarelada selecionou-se o agrupamento de menor valor para a Equação 1 no espaço RGB.

$\max (R)-\max (G)$

A fim de refinar a detecção de exsudatos são geradas duas novas imagens $I_{3}$ e $I_{4}$. A primeira é formada por todas as regiões de $I_{2}$ presentes em $I_{1} \mathrm{e}$ a segunda é formada pelas demais regiões de $I_{l}$ não presentes em $I_{2}$.

\section{Remoção de falsos candidatos}

É possível observar a existência de partes do DO e de regiões brilhantes da retina marcadas como exsudatos, mesmo após realizadas todas as etapas anteriores (a Figura 8f apresenta um resultado semelhante ao do algoritmo de Ram e Sivaswamy). A principal característica dessas regiões destacadas é que estas são limitadas por vasos sanguíneos, os quais devem ser identificados e removidos, nesta aplicação proposta. No algoritmo original esta remoção é realizada por alargamento de contraste, que tem o objetivo de melhorar o contraste da imagem. Para isto, uma faixa de níveis de cinza tem seu valor incrementado, enquanto duas outras faixas de níveis de cinza têm seus valores decrementados (Gonzalez e Woods, 2010).

Após a operação de agrupamento, a região de vasos apresenta os mais altos valores da componente R, enquanto que as áreas de exsudatos e regiões brilhantes apresentam os mais altos valores da componente G. Dessa forma, se a média dos valores dos pixels de cada objeto presente, na componente $\mathrm{R}$, for maior que a média desses objetos na imagem original, então o mesmo é removido. Espera-se, assim, que os objetos pertencentes à região do $\mathrm{DO}$ sejam eliminados, pois é nessa região que se encontram os vasos mais calibrosos da retina. Ao término desse processo, as duas imagens resultantes são limiarizadas utilizando o método de Otsu (Chutatape e Dawson, 1996). A junção dessas duas imagens constitui o resultado final do algoritmo introduzido por Ram e Sivaswamy (2009), ou seja, os objetos presentes nessa imagem são candidatos a exsudatos. Importante notar que em algumas situações o processo de limiarização elimina todas as regiões, indicando assim que a imagem original é saudável, ou seja, não contém exsudatos.

\section{Método proposto}

A partir da análise dos resultados do algoritmo original, foram observados pixels isolados e regiões pertencentes ao DO, que foram classificados como exsudatos. Esse fato pode ser consequência de alguma falha no processo de agrupamento ou na remoção de falsos candidatos. Para corrigir esse problema, foram introduzidas modificações nesse algoritmo, destacados em azul, como ilustra o diagrama da Figura 4.

\section{Agrupamento de pixels}

No algoritmo $K$-médias um objeto pertence a um único grupo apenas, enquanto que nas técnicas de agrupamentos nebulosos (fuzzy clustering), os dados podem possuir características que permitam associá-los a diversos grupos, segundo uma função de pertinência ponderada (Bezdek et al., 1993). Esse interessante aspecto destaca que um determinado indivíduo não é típico de um grupo específico e que se assemelha em maior ou menor grau com indivíduos de mais de um grupo. Dessa forma, o algoritmo $K$-médias nebuloso se mostra menos sensível a erros de agrupamento, causados pela diferença nos padrões de iluminação da imagem. De fato, este algoritmo foi integrado a outros trabalhos de detecção de exsudatos (Osareh et al., 2001, 2009; Sopharak et al., 2009; Zhang e Chutatape, 2005), sendo que alguns destes métodos foram utilizados no presente artigo para comparação e avaliação de desempenho do método proposto.

Nos testes preliminares dos algoritmos propostos foram adotados os mesmos parâmetros de ajuste do algoritmo $K$-médias nebuloso apresentados no experimento de Sopharak et al. (2009), a saber, grau de nebulosidade igual 2, número de iterações 200 e

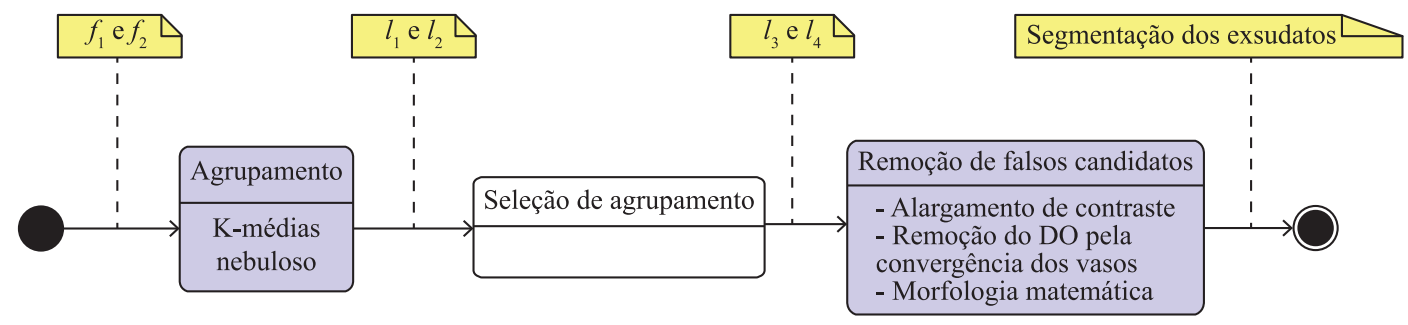

Figura 4. Diagrama do sistema proposto. Regiões em azul representam as etapas modificadas.

Figure 4. Diagram of the proposed system. Blue regions represent the modified steps of the original algorithm. 
erro máximo de $10^{-6}$. Posteriormente, variações destes parâmetros foram testadas com o objetivo de ajustar valores para os quais os resultados de agrupamento não se alterassem com os mesmos. Assim, o grau de nebulosidade utilizado foi mantido em 2, a quantidade máxima de iterações foi fixada em 2000 e o limiar de distorção máximo em $10^{-8}$. Ressalta-se que a escolha empírica desses valores garantiu que a aleatoriedade da escolha dos agrupamentos iniciais não interferisse no resultado final do agrupamento. Assim, o algoritmo proposto converge sempre para o mesmo resultado, independente da escolha dos grupos iniciais.

\section{Remoção de falsos candidatos}

Outra contribuição deste artigo consiste na proposta da eliminação do DO através da detecção dos vasos sanguíneos. Essa técnica foi adotada, porque estratégias que utilizam a informação de que o DO é a região de convergência dos vasos têm sido bem sucedidas para este fim. Para a detecção dos vasos sanguíneos foi utilizado um algoritmo baseado em técnicas de MM proposto por Zana e Klein (2001). Neste algoritmo, a rede de vasos foi representada por segmentos de retas com a aplicação da transformada de Hough (Gonzalez e Woods, 2010) e foi realizada uma busca por três janelas quadradas de lado igual à metade do raio do DO (70 pixels) com a maior quantidade e proximidade de retas. $\mathrm{O}$ centro do DO foi determinado como sendo o centro da janela que apresentar maior quantidade de pixels brancos na imagem de vasos. A justificativa desta escolha se deve ao fato de que os vasos que convergem para o DO são os mais calibrosos da rede.

A eliminação do DO foi realizada de duas formas, sendo que em uma delas foi removida uma janela de tamanho aproximadamente igual ao DO, enquanto que na outra foi removida a região conectada ao DO. A eliminação da região é bem específica e remove apenas partes conectadas à região identificada como $\mathrm{DO}$, enquanto que a remoção da janela elimina regiões da vizinhança do DO.

Para eliminação de regiões com falsos candidatos foram avaliadas técnicas que utilizam morfologia matemática. A MM pode ser caracterizada como um conjunto específico de operações sobre imagens capaz de analisar estruturas espaciais, extrair informações relativas à geometria e à topologia de uma imagem, através de um conjunto completamente definido, denominado Elemento Estruturante (Soille, 2003). Neste artigo, optamos pela utilização de uma abertura morfológica a qual é composta de uma erosão seguida de uma dilatação. A abertura geralmente suaviza o contorno de um objeto, rompe os istmos e elimina as saliências finas. Quanto à escolha da forma e tamanho do elemento estruturante, tomamos por base o padrão dos falsos candidatos, os quais são regiões pequenas e de formato circular. Assim, as simulações realizadas levaram à escolha de um elemento estruturante na forma de uma circunferência de raio igual a 3 pixels.

\section{Resultados}

Na literatura, a avaliação dos resultados de algoritmos de detecção de exsudatos é realizada de três formas: com base na imagem, em regiões, ou, mais especificamente, pixel a pixel. Neste trabalho, foram utilizadas apenas as avaliações por imagens e por regiões, pois a base de testes DIARETDB1 contém marcações dos exsudatos para as mesmas, enquanto que a avaliação pixel a pixel, não está disponível para a referida base.

A avaliação por imagens consiste em verificar se cada imagem foi classificada corretamente como saudável (não possui exsudatos) ou patológica (possui exsudatos). $\mathrm{Na}$ avaliação por regiões, quando os candidatos a exsudatos encontrados pelo algoritmo corresponderem a pelo menos $50 \%$ de uma região marcada pelos especialistas, então o resultado é validado para toda região. Esse critério foi utilizado por Ram e Sivaswamy (2009), e se fundamenta no fato de que os exsudatos são pequenos, apresentam forma irregular e aparecem em grupos.

Para avaliar o desempenho dos algoritmos foram ainda utilizadas as métricas denominadas Sensibilidade, Especificidade, Verdadeiro Preditivo Positivo (VPP), Verdadeiro Preditivo Negativo (VPN) e Acurácia. Todas essas métricas são calculadas com base nos valores de Verdadeiro Positivo (VP), Falso Positivo (FP), Verdadeiro Negativo (VN) e Falso Negativo (FP). A Tabela 1 apresenta o significado e a forma de calcular todas essas medidas.

Dentre as 53 imagens saudáveis disponíveis na base utilizada, o algoritmo original de Ram e Sivaswamy (2009) classificou 42 incorretamente, ou seja, classificou as imagens saudáveis como patológicas em $80 \%$ dos casos, o que resultou em apenas 52,81\% de Acurácia. Neste artigo, o algoritmo modificado, por uso combinado do algoritmo $K$-médias nebuloso foi denominado Fuzzycluster 1. Os resultados da avaliação dos experimentos realizados com a versão original introduzida por Ram e Sivaswamy (2009) e a proposta, neste artigo, para detecção de exsudatos são mostrados no gráfico de colunas da Figura 5.

Analisando os resultados da Figura 5, observa-se que o algoritmo original apresenta a medida de Acurácia equivalente à obtida pelo algoritmo Fuzzycluster 1 com relação à avaliação por imagens, ou seja, $52,81 \%$ e $49,44 \%$, respectivamente. Por outro lado, observa-se que para a avaliação por regiões, a medida de Acurácia obtida do algoritmo Fuzzycluster 1 foi maior que a do algoritmo original, ou seja, $78,35 \%$ e $73,90 \%$, respectivamente. Esta 
melhoria de desempenho na detecção, decorrente do uso da técnica de agrupamento $K$-médias nebuloso, mostrou que o algoritmo Fuzzycluster 1 foi capaz de diferenciar melhor as lesões brilhantes de outras regiões presentes na sua vizinhança. Com isso, os candidatos a exsudatos resultantes apresentaram contornos similares aos dos exsudatos marcados pelos especialistas, ou padrão ouro.

Tabela 1. Medidas de avaliação de desempenho.

Table 1. Performance evaluation measures.

\begin{tabular}{|c|c|c|}
\hline Medidas & Classificação por imagens & Classificação por regiões \\
\hline Verdadeiro Positivo (VP) & $\begin{array}{l}\text { Quantidade de imagens classificadas } \\
\text { corretamente como saudáveis. }\end{array}$ & $\begin{array}{l}\text { Quantidade de pixels classificados } \\
\text { corretamente como saudáveis. }\end{array}$ \\
\hline Falso Positivo (FP) & $\begin{array}{l}\text { Quantidade de imagens classificadas } \\
\text { incorretamente como saudáveis. }\end{array}$ & $\begin{array}{l}\text { Quantidade de pixels classificados } \\
\text { incorretamente como saudáveis. }\end{array}$ \\
\hline Verdadeiro Negativo (VN) & $\begin{array}{l}\text { Quantidade de imagens classificadas } \\
\text { corretamente como patológicas. }\end{array}$ & $\begin{array}{l}\text { Quantidade de pixels classificados } \\
\text { corretamente como patológicas. }\end{array}$ \\
\hline \multirow[t]{2}{*}{ Falso Negativo (FN) } & $\begin{array}{l}\text { Quantidade de imagens classificadas } \\
\text { incorretamente como patológicas. }\end{array}$ & $\begin{array}{l}\text { Quantidade de pixels classificados } \\
\text { incorretamente como patológicas. }\end{array}$ \\
\hline & Fórmula & Significado \\
\hline Sensibilidade & $S=\frac{V P}{V P+F N}$ & Proporção de verdadeiros positivos. \\
\hline Especificidade & $E=\frac{V N}{V N+F P}$ & Proporção de verdadeiros negativos. \\
\hline $\begin{array}{l}\text { Verdadeiro Preditivo } \\
\text { Positivo (VPP) }\end{array}$ & $V P P=\frac{V P}{V P+F P}$ & $\begin{array}{l}\text { Proporção de verdadeiros positivos em } \\
\text { relação a todas as predições positivas. }\end{array}$ \\
\hline $\begin{array}{l}\text { Verdadeiro Preditivo } \\
\text { Negativo (VPN) }\end{array}$ & $V P N=\frac{V N}{V N+F N}$ & $\begin{array}{l}\text { Proporção de verdadeiros negativos em } \\
\text { relação a todas as predições negativas. }\end{array}$ \\
\hline Acurácia & $A=\frac{V P+V N}{V P+F P+F N+V N}$ & $\begin{array}{c}\text { Proporção de predições corretas, ou seja, o } \\
\text { acerto total. }\end{array}$ \\
\hline
\end{tabular}
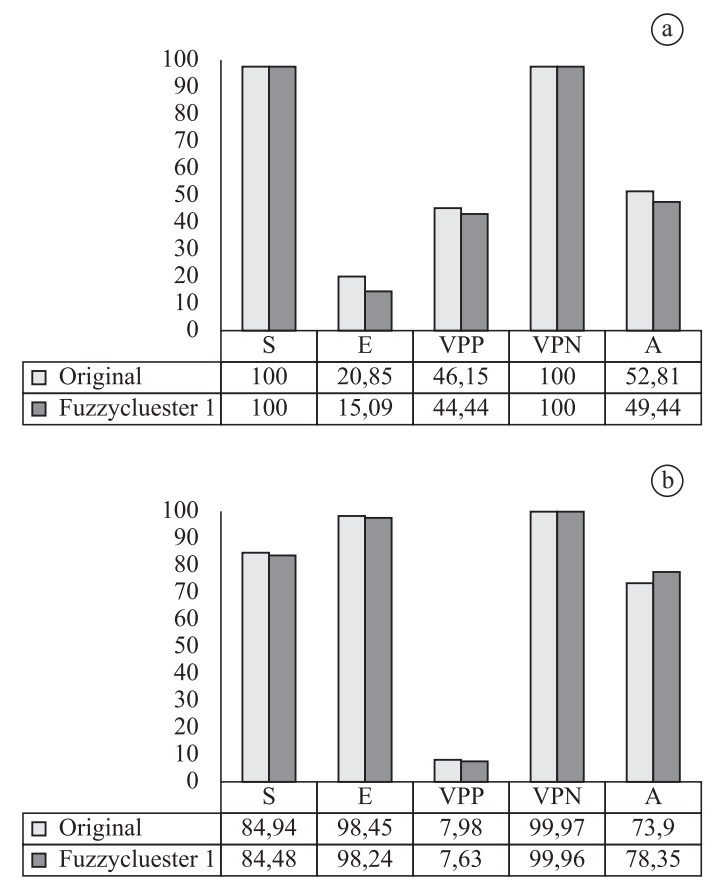

Figura 5. Avaliação de desempenho, em porcentagem, dos algoritmos original e Fuzzycluster 1: a) por imagem e b) por regiões. Figure 5. Performance evaluation (percentage) of the original algorithm and Fuzzycluster 1: a) by image and b) by regions. 
Outra contribuição deste artigo foi a integração de um módulo de detecção do DO à metodologia proposta de detecção de exsudatos, considerando que o DO é uma região de convergência dos vasos sanguíneos e que contém falsos candidatos associados. A partir desta integração, os novos algoritmos foram denominados como segue: a) Fuzzycluster 2 - algoritmo que integra os módulos de agrupamento ( $K$-médias nebuloso) e remoção da janela do DO (RJDO) pela detecção dos vasos; b) Fuzzycluster 3 - algoritmo que integra os módulos de agrupamento ( $K$-médias nebuloso) e remoção da região conectada ao DO (RRDO). Os resultados desses algoritmos são mostrados nos gráficos da Figura 6a, que apresenta a avaliação de desempenho por imagens, e na Figura $6 \mathrm{~b}$ a avaliação de desempenho por regiões.

Analisando os resultados apresentados na Figura 6, observa-se que 0 algoritmo que obteve melhor resultado na avaliação por imagens foi o Fuzzycluster $2 \mathrm{com}$ Acurácia de $73,03 \%$, enquanto na avaliação por regiões foi o Fuzzycluster 3, com Acurácia de 99,25\%. O melhor desempenho por avaliação de imagens foi do algoritmo que integra a remoção do DO e isso se justifica pelo fato de que a remoção de toda a janela engloba pixels isolados próximos ao DO. Por outro lado, a remoção da região conectada ao DO elimina apenas as regiões que realmente pertencem ao disco óptico. Essa tendência não se verifica na avaliação por região, porque pixels isolados contribuem muito pouco para a taxa de erro, quando comparamos esta quantidade de pixels ao total presente na imagem.

Mesmo com a melhoria resultante da eliminação do DO, observou-se que ainda assim permaneciam regiões candidatas e que as mesmas se caracterizavam por apresentarem pixels isolados na imagem. Com o intuito de reduzir a quantidade de falsos candidatos remanescentes, optou-se pelo pós-processamento da imagem segmentada utilizando uma abertura morfológica (AM). Em consequência desta nova etapa de processamento, denominamos de Fuzzycluster 4 o algoritmo resultante da combinação do algoritmo Fuzzycluster 2 com a operação de AM. A denominação Fuzzycluster 5 foi associada à combinação do algoritmo Fuzzycluster 3 com a operação de AM. Para avaliar a robustez dos algoritmos, foram realizados experimentos com 4 grupos de imagens de retina oriundos da base de testes, sendo cada grupo composto com igual número de imagens doentes e saudáveis. A partir

\section{(a)}

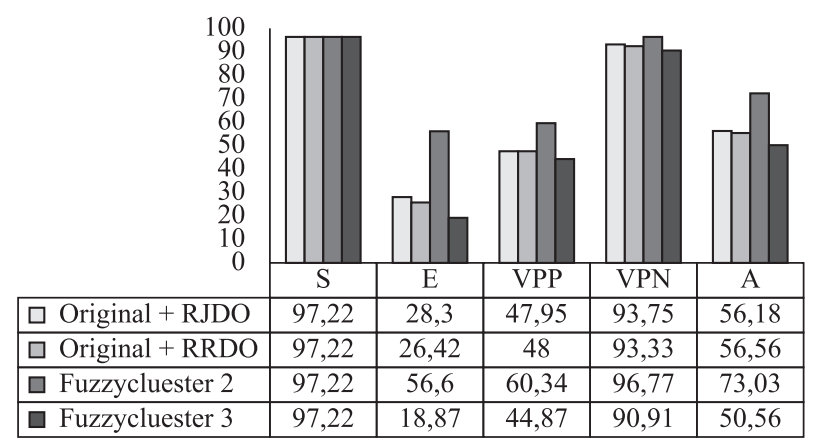

(b)

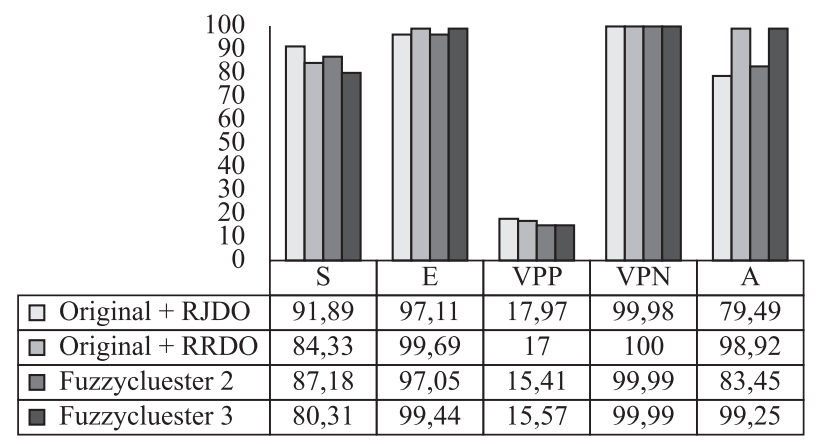

Figura 6. Avaliação de desempenho, em porcentagem, dos algoritmos original com remoção da janela e região do DO, Fuzzycluster 2 e Fuzzycluster 3: a) por imagem e b) por regiões.

Figure 6. Performance evaluation (percentage) of the original by removing the window and DO region and Fuzzycluster 2 and Fuzzycluster 3 algorithms: a) by image and b) by regions. 
destes resultados de avaliação, foram levantadas medidas estatísticas, seus valores máximos, mínimos e desvio padrão. A Tabela 2 exibe estes resultados de avaliação e a Figura 7 apresenta os valores médios, máximos e mínimos dessas medidas.

\section{Discussão}

As Figuras 6 e 7 exibem os resultados dos experimentos com os algoritmos Fuzzycluster 2 e 4 e mostra que os mesmos apresentaram os melhores desempenhos, pela avaliação por imagens, com Acurácia igual a 73,03\%. Com relação à avaliação de regiões, o algoritmo que obteve melhor desempenho foi o Fuzzycluster 5 , com Acurácia igual a $99,41 \%$. A melhoria do desempenho com base em regiões foi proporcionada pela eliminação, através da operação de AM, dos pixels isolados. A despeito da aplicação desta operação, falsos candidatos a exsudatos ainda permaneceram, e em consequência as avaliações de desempenho por regiões e por imagens se assemelharam.

Tabela 2. Avaliação de desempenho dos algoritmos Fuzzycluster 4 e Fuzzycluster 5.

Table 2. Performance evaluation of Fuzzycluster 4 and Fuzzycluster 5 algorithms.

\begin{tabular}{|c|c|c|c|c|c|c|c|c|c|}
\hline \multirow[b]{2}{*}{ Algoritmo } & \multirow[b]{2}{*}{ Taxa } & \multicolumn{4}{|c|}{ Avaliação por imagem } & \multicolumn{4}{|c|}{ Avaliações por região } \\
\hline & & $\begin{array}{c}\text { Média } \\
(\%)\end{array}$ & $\begin{array}{l}\text { Máx } \\
(\%)\end{array}$ & $\begin{array}{l}\text { Min } \\
(\%)\end{array}$ & $\begin{array}{c}\text { Desvio } \\
(\%)\end{array}$ & $\begin{array}{c}\text { Média } \\
(\%)\end{array}$ & $\begin{array}{c}\text { Máx } \\
(\%)\end{array}$ & $\begin{array}{l}\text { Min } \\
(\%)\end{array}$ & $\begin{array}{c}\text { Desvio } \\
(\%)\end{array}$ \\
\hline \multirow{5}{*}{ Fuzzycluster 4} & Sensibilidade & 100 & 100 & 100 & 0 & 82,26 & 85,65 & 74,50 & 0,51 \\
\hline & Especificidade & 54,24 & 69,23 & 30,77 & 0,53 & 97,14 & 99,15 & 95,05 & 0,2 \\
\hline & VPP & 60,55 & 69,23 & 50,00 & 0,8 & 16,42 & 24,31 & 7,07 & 0,71 \\
\hline & VPN & 100 & 100 & 100 & 0 & 99,99 & 100 & 99,97 & 0,001 \\
\hline & Acurácia & 72,73 & 81,82 & 59,09 & 0,97 & 91,23 & 99,67 & 83,76 & 0,68 \\
\hline \multirow{5}{*}{ Fuzzycluster 5} & Sensibilidade & 94,28 & 100 & 88,24 & 0,66 & 99,50 & 99,76 & 98,81 & 0,8 \\
\hline & Especificidade & 30,56 & 46,15 & 22,22 & 1,10 & 77,55 & 99,76 & 66,04 & 0,004 \\
\hline & VPP & 48,09 & 56,25 & 41,67 & 0,6 & 18,57 & 24,58 & 14,25 & 0,46 \\
\hline & VPN & 88,75 & 100,00 & 75,00 & 1,73 & 99,99 & 100 & 99,97 & 0,0001 \\
\hline & Acurácia & 56,25 & 68,18 & 47,73 & 0,86 & 99,73 & 100 & 99,41 & 0,003 \\
\hline
\end{tabular}
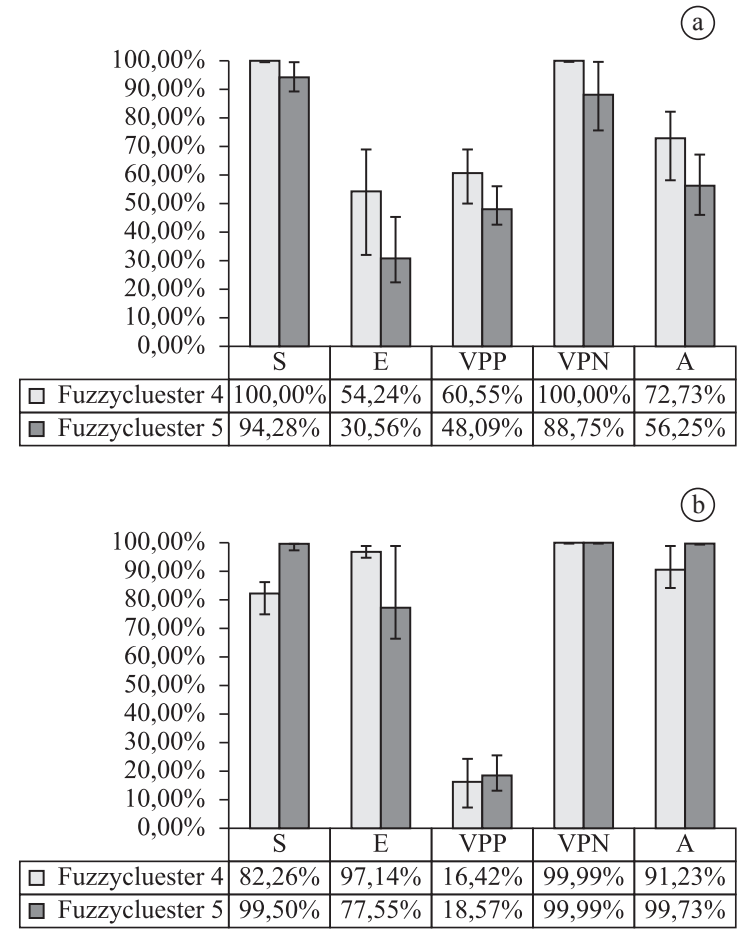

Figura 7. Avaliação de desempenho dos algoritmos Fuzzycluster 4 e 5: a) por imagem e b) por regiões.

Figure 7. Performance evaluation of the Fuzzycluster 4 and 5 algorithms: a) by image and b) by regions. 

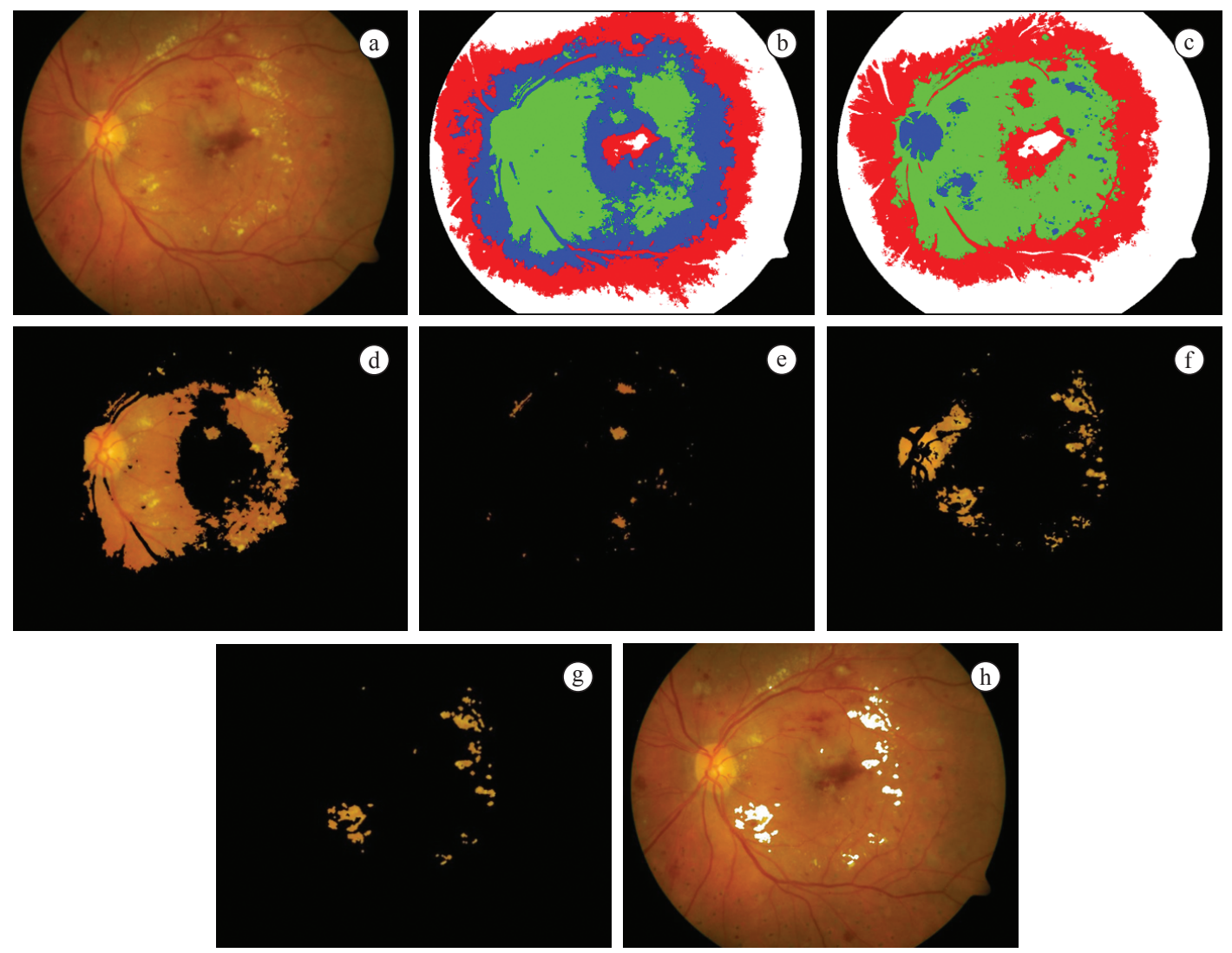

Figura 8. Aplicação do algoritmo Fuzzycluster 5: a) imagem original, b) agrupamento com o vetor $\mathrm{f}_{1}$ (imagem $\mathrm{I}_{1}$ ), c) agrupamento com o vetor $\mathrm{f}_{2}\left(\right.$ imagem $\mathrm{I}_{2}$ ), d) seleção dos grupos em $\mathrm{I}_{1}$ (imagem $\mathrm{I}_{1} \cdot 3$ ), e) seleção dos grupos em $\mathrm{I}_{2}$ (imagem $\mathrm{I}_{2} \cdot 3$ ), f) resultado final da seleção das regiões candidatas a exsudatos nas imagens $\mathrm{I}_{1} .3$ e $\mathrm{I}_{2} .3, \mathrm{~g}$ ) regiões classificadas como exsudatos após a eliminação dos falsos candidatos, h) sobreposição do resultado na imagem original.

Figure 8. Application of the Fuzzycluster 5 algorithm: a) original image, b) clustering result with the vector $f_{l}\left(I_{l}\right.$ image), c) clustering result with the vector $f_{2}\left(I_{2}\right.$ image), d) group selection in $I_{I}$ (image $\left.I_{l} .3\right)$, e) group selection in $I_{2}$ (image $\left.I_{2} \cdot 3\right)$, f) final selection result of the candidate exudate regions in images $I_{I}, 3$ and $I_{2}, 3, g$ ) regions classified as exudates after the elimination of false candidates, $h$ ) overlaid the result on the original image.

Na fase de desenvolvimento e testes da metodologia apresentada, foram realizados vários experimentos morfológicos e a operação de AM com elemento estruturante circular, de raio igual a 3 pixels, apresentou a melhor escolha para ambas avaliações, isto é, por imagem e por região. Como exemplo, destacamos o caso em que a utilização de elementos estruturantes maiores (com até 7 pixels de raio) melhorou a Acurácia do algoritmo na avaliação por imagens, atingindo uma taxa de $93 \%$ de acerto. Porém, o desempenho com base nas regiões alcançou apenas $62 \%$.

A Figura 8 apresenta o resultado da aplicação do algoritmo Fuzzycluster 5. Analisando o desempenho de algoritmos que foram avaliados com o mesmo critério, o referido algoritmo apresentou melhor desempenho do que o proposto por Ram e Sivaswamy (2009) e as técnicas introduzidas por Osareh et al. (2001) e Sopharak et al. (2009). Estes últimos não utilizaram imagens de bases públicas, e realizaram seus testes com imagens de hospitais locais. Em Osareh et al. (2001) foi relatada uma taxa de Acurácia de 92\% obtida em 42 imagens, enquanto em Sopharak et al. (2009) a taxa de acerto alcançada foi de $87,28 \% \mathrm{em}$ 40 imagens.

\section{Conclusão}

Neste artigo foi apresentada uma metodologia de detecção de exsudatos em imagens de retina da base DIARETDB1, que combina agrupamento de pixels e remoção de falsos candidatos. Trata-se, portanto, de uma proposta de localização e segmentação de exsudatos que utiliza agrupamento nebuloso, morfologia matemática e detecção do DO na remoção de falsos candidatos com o objetivo de alcançar maiores taxas da medida Acurácia, nesta base de testes.

A partir dos resultados obtidos conclui-se neste trabalho, que o sucesso alcançado pela metodologia 
estendida se deve à inclusão de um algoritmo de detecção do DO, para fins de eliminação de falsos candidatos que estejam nesta região e na sua vizinhança. A proposta adotada para a localização da região do DO se fundamenta no fato do mesmo ser uma região de convergência dos vasos e que os exsudatos possuem propriedades de cor e forma semelhantes às desta região. Com isso, algoritmos disponíveis na literatura, baseados em técnicas de agrupamento, limiarização, crescimento de região e reconstrução morfológica, que consideram apenas essas características não distinguem eficazmente exsudatos de falsos candidatos.

Com a remoção do DO, foram eliminados falsos candidatos remanescentes através da aplicação bem sucedida de um pós-processamento com operação de abertura morfológica. Como avaliação final, os testes mostraram que o algoritmo resultante deste trabalho é confiável e adequado para uso em sistemas automáticos de avaliação de imagens de pacientes com retinopatia diabética. Por se tratar de um método de deteç̧ão de exsudatos, o mesmo pode auxiliar o especialista na graduação de doenças como o edema macular, segundo o posicionamento dos mesmos em relação à região macular. Ademais, concluímos que o resultado da etapa de agrupamento dos dados em múltiplos espaços de cores mostrou-se eficaz na segmentação de outras estruturas da retina como a mácula e o próprio DO.

Para trabalhos futuros sugerimos o estudo de técnicas de extração e análise dos atributos das regiões candidatas a exsudatos e sua utilização em algoritmos de classificação (ex. MLP, SVM) para fins de remoção de falsos candidatos.

\section{Agradecimentos}

Ao Laboratório de Inteligência Computacional - LabInC da Universidade Federal do Piauí, ao Laboratório de Visão, Imagens e Sinais - LabVis da Universidade Federal do Ceará e à Fundação Cearense de Apoio ao Desenvolvimento Científico e Tecnológico - FUNCAP.

\section{Referências}

Basha S, Prasad K. Automatic detection of hard exudates in diabetic retinopathy using morphological segmentation and fuzzy logic. International Journal of Computer Science and Network Security. 2008; 8(12):211-8.

Bezdek JC, Hal LO, Clarke LP. Review of MRI images segmentation techniques using pattern recognition. Medical Physics. 1993; 20(4):1033-48. PMid:8413011. http://dx.doi. org/10.1118/1.597000

Chutatape O, Dawson BA. Thresholding method for blood aggregate images. IEEE Engineering in
Medicine and Biology. 1996; 15(3):103-8. http://dx.doi org/10.1109/51.499767

García M, Sánchez CI, López MI, Abasólo D, Hornero R. Neural network based detection of hard exudates in retinal images. Computer Methods and Programs in Biomedicine. 2009; 93(1):9-19. PMid:18778869. http:// dx.doi.org/10.1016/j.cmpb.2008.07.006

Giancardo L, Meriaudeau F, Karnowski T, Li Y, Garg S, Tobin Junior K, Chaum E. Exudate-based diabetic macular edema detection in fundus images using publicly available datasets. Medicine Image Analysis. 2012; 16(1):216-26. PMid:21865074. http://dx.doi.org/10.1016/j. media.2011.07.004

Gonzalez RC, Woods RE. Processamento digital de imagens. 3. ed. São Paulo; 2010.

Katarzyna S, Adam S, Radim C, Georg M. Segmentation of fundus eye images using methods of mathematical morphology for glaucoma diagnosis. Lecture Notes in Computer Science. 2004; 3039:41-8. http://dx.doi. org/10.1007/978-3-540-25944-2_6

Kauppi T, Kalesnykiene V, Kamarainen JK, Lensu L, Sorri I, Kälviäinen H, Pietilä J. The Diaretdb1 diabetic retinopathy database and evaluation protocol. In: Conference on Medical Image Understanding and Analysis: Proceedings of the 11th Conference on Medical Image Understanding and Analysis; 2007; Aberystwyth, Gales. Aberystwyth; 2007. p. 252-61.

Köse C, Sevik U, Ikibas C, Erdöl H. Simple methods for segmentation and measurement of diabetic retinopathy lesions in retinal fundus images. Computer Methods and Programs in Biomedicine. 2012; 107(2):274-93. PMid:21757250. http://dx.doi.org/10.1016/j.cmpb.2011.06.007

Kavitha D, Shenbaga SD. Automatic detection of optic disc and exudates in retinal images. In: ICISIP 2005: Proceedings of the International Conference on Intelligent Sensing and Information Processing; 2005; Madras, India. Madras; 2005. p. 501-6. http://dx.doi.org/10.1109/ICISIP.2005.1529506

Osareh A, Mirmehdi M, Thomas B, Markham R. Automatic recognition of exudative maculopathy using fuzzy C-Means clustering and neural networks. In: Medical Image Understanding and Analysis Conference: Proceedings of the Medical Image Understanding and Analysis Conference; 2001; Birmingham, Reino Unido. Birmingham; 2001. p. 49-52.

Osareh A, Shadgar B, Markham R. A computationalintelligence-based approach for detection of exudates in diabetic retinopathy images. IEEE Transactions on Information Technology in Biomedicine. 2009; 13(9):535-45. PMid:19586814. http://dx.doi.org/10.1109/ TITB.2008.2007493

Ram K, Sivaswamy J. Multi-space clustering for segmentation of exudates in retinal color photographs. In: Annual International Conference of the IEEE Engineering in Medicine and Biology: Proceedings of the Annual International Conference of the IEEE Engineering in Medicine and Biology; 2009; Minessota, EUA. Minessota; 2009. 
p. 1437-40. PMid:19963747. http://dx.doi.org/10.1109/ IEMBS.2009.5332911

Sinthanayothin C, Boyce JF, Williamson TH, Cook HL, Mensah E, Lal S, Usher D. Automated detection of diabetic retinopathy on digital fundus images. Diabetic Medicine 2002; 19(2):105-12. PMid:11874425. http:// dx.doi.org/10.1046/j.1464-5491.2002.00613.x

Soille P. Morphological image analysis: Principles and applications. 2. ed. Nova York: Springer; 2003.

Sopharak A, Uyyanonvara B, Barman S. Automatic exudates detection from non-dilated diabetic retinopathy retinal images using c-means clustering. Sensors. 2009; 9(3):2148-61. PMid:22574005 PMCid:3332251. http://dx.doi.org/10.3390/ s90302148

Sopharak A, Dailey MN, Uyyanonvara B, Barman S, Williamson T, Nwe TN, Moe AY. Machine learning approach to automatic exudate detection in retinal images from diabetic patients. Journal of Modern Optics. 2010; 57:124-35. http:// dx.doi.org/10.1080/09500340903118517
Usher D, Dumskyj M, Himaga M, Williamson TH, Nussey $\mathrm{S}$, Boyce JJ. Automated detection of diabetic retinopathy in digital retina images: a tool for diabetic retinopathy screening. Diabetic Medicine. 2004; 21(1):84-90. PMid:14706060. http://dx.doi.org/10.1046/j.1464-5491.2003.01085.x

Walter T, Klevin J, Massin P, Erginay A. A contribution of image processing to the diagnosis of diabetic retinopathy-detection of exudates in color fundus images of the human retina. IEEE Transactions on Medical Imaging. 2002; 21(10):1236-43. PMid:12585705. http:// dx.doi.org/10.1109/TMI.2002.806290

Zana F, Klein JC. Segmentation of vessel-like patterns using mathematical morphology and curvature evaluation. IEEE Transactions on Image Processing. 2001; 10(7):1010-19. PMid:18249674. http://dx.doi.org/10.1109/83.931095

Zhang X, Chutatape O. Top-down and bottom-up strategies in lesion detection of background diabetic retinopathy. In: Conference on Computer Vision and Pattern Recognition: Proceedings of the Conference on Computer Vision and Pattern Recognition; 2005; San Diego, EUA. San Diego; 2005. p. 422-8.

\footnotetext{
Autores

Rodrigo de Melo Souza Veras*

Departamento de Computação, Universidade Federal do Piauí - UFPI, Teresina, PI, Brasil.

Departamento de Engenharia de Teleinformática, Universidade Federal do Ceará - UFC, Fortaleza, CE, Brasil.
}

Flávio Henrique Duarte de Araújo, André Macêdo Santana, Romuere Rodrigues Veloso e Silva

Departamento de Computação, Universidade Federal do Piauí - UFPI, Teresina, PI, Brasil.

Fátima Nelsizeuma Sombra de Medeiros

Departamento de Engenharia de Teleinformática, Universidade Federal do Ceará - UFC, Fortaleza, CE, Brasil. 